\title{
Archaea and their potential pathogenicity in human and animal diseases
}

\section{Review Article}

Volume: 3, Issue: 3 December 2019 Pages: $79-84$

\author{
Aikerim Kumondorova ${ }^{1}$, Serkan íkiz ${ }^{2}$ \\ 1.Istanbul University-Cerrahpasa, Institute of Graduate Studies, 34320, Avcilar/ Istanbul, Turkey \\ 2.Istanbul University-Cerrahpasa, Faculty of Veterinary Medicine, Department of Microbiology, \\ Buyukcekmece Campus 34500, Istanbul, Turkey, \\ Kumandorova A.: ORCID: 0000-0001-7341-1597; İkiz S.: ORCID: 0000-0001-6502-0780
}

\section{Article History}

Received: 11.11 .2019

Accepted: 20.12.2019

Available online:

24.12.2019

\begin{abstract}
There are hundreds of organisms that infect and cause disease in humans and animals. These organisms can be bacteria and single-celled eukaryote, as well as a few parasites. Archaea, one of the three domain of life, immensely diverse group of prokaryotes and includes a number of "extremophiles" that develop in such environments as hot springs, salt lakes, human and animal gut, volcanic submarines and low, high $\mathrm{pH}$ habitats. It is puzzling that despite being one of the most numerous and ubiquitous life forms on earth, no member of the domain Archaea has been described as human or animal pathogen. The absence of pathogenic Archaea in the taxonomy database is statistically highly significant. The aim of this article is to display a brief overview of what is currently known about archaea and archaeal potential pathogenicity in and on human being and animals.
\end{abstract}

Keywords: Archaea, pathogenicity, disease, human, animal

DOI: 10.30704/http-www-jivs-net.641538

To cite this article: Kumondorova, A., \& ikiz, S. (2019). Archaea and their potential pathogenicity in human and animal diseases. Journal of Istanbul Veterinary Sciences. 3(3), 79-84, Abbreviated Title: J Ist Vet Sci

\section{Introduction}

Archaeabacteria are single-celled organisms that can survive in extreme conditions and they have challenged the way scientists classify life. They are believed to be the oldest form of organisms, being about 3.5 billion years old. In the past, they were placed under the Kingdom monera along with bacteria. However, this classification is no longer followed. Since Archaeabacteria are biochemically and genetically different from bacteria and possess unique evolutionary history, they have a separate domain in the three-domain system of biological classification. In fact, Archaeabacteria are no longer called so, they are instead known as Archaea. The term achaio is a Greek word, which means 'ancient'. The meaning of the word aptly describes the Archaeabacteria who are thought to have a common ancestor like the bacteria and eukaryotes. Archaeabacteria is similar in structure (biochemical and genetic features) to eukaryotes than bacteria (Balch et al., 1979; Madigan et al., 2000).

\section{The First Findings}

Several scientist groups and institutions have not yet to find powerful evidence of an Archaeal pathogen, although some Archaeal phenotypes inhabit the human body and share commensal and symbiotic relationships with many species of animals and single-celled eukaryotes. Several theories have been published

*Corresponding Author: Aikerim Kumandorova

E-mail: akumondorova@gmail.com 
according to Archaea and their relationship to diseases. Since the publication of the Cavicchioli's (2003) article, meta-genomic studies have revealed more about the diversity of microbial life and prevalence of Archaea domain (Cavicchioli et al., 2003; Eckburg et al., 2003).

According to Cavicchioli et al. (2003) they are (Archaea) a highly diverse domain of life that is present in highly numbers in the environment that would afford them the opportunity to cause disease. They are able to interact with eukaryotic cells in symbiotic relationships, suggesting that pathogenic relationships may be possible. Archaea subspecies are present in many animals, especially in ruminants 'digest system or in human oral cavity, vagina, and they are recognized by the immune system (Cavicchioli et al. 2003). Cavicchioli et al. (2003) have written that it is likely that there are archaeal pathogens, but they have not yet been discovered. So far, as the publication of the Cavicchioli et al. (2003) released, meta-genomic studies have revealed more about the diversity of microbial life and prevalence of Archaea species. Unfortunately, such methods are worthless tools for illuminating the causes of unknown illnesses (Cavicchioli et al., 2003).

Another researcher Martin (2004) postulates that, "There are two possible reasons why no pathogenic archaea are known. First, they do not exist or second is they have not been identified yet". He also wrote that, Archaea are not pathogens because they use different co-factors in their biochemical reactions compared to Eukarya and Bacteria. For instance, vitamins like co-enzyme $M$, cobamides, factor F430, co-enzyme B. That is why, Archaea do not parasitize since they have enough food to survive. As how we know, pathogens are looking for a meal for infection and growth (Martin, 2004).

\section{Archaea at the present}

Moreover, Gill and Brinkman (2011) published different hypothesis that Archaea may contribute to disease caused by other organisms indirectly. They presume Archaea may facilitate the growth of diseasecausing organisms rather than causing disease directly by themselves. It can be possible by removing $\mathrm{H} 2$ from microbiota where complex microbial communities exist. $\mathrm{H} 2$ inhibits the growth of some disease-causing bacteria, and once removed, these species could flourish. In addition to that, it has known that methanogenic Archaea has been connected with various human disease, such as periodontal disease, gastrointestinal ailments, and colon cancer; however no causative relationships have been established exactly. For these reasons, this hypothesis requires further study and to be confirmed.

Also it was assumed that disease causing bacteria may have receive some of their virulence factors from Archaea through the transfer of "Pathogenic" genes from species that engage in symbiotic or commensal relationships with Eukarya. Nevertheless, gene transfer has not been inferred between Bacteria and Archaea exactly until today and the mechanisms that provide the transfer have not been classified yet. Currently anyone definitively pointed pathogenesis with any gene that has been transferred from Archaea to a bacterium. Furthermore, there is hypothesized that virulence bacteriophages could not interact with Archaea, in this way hindering the ability of Archaea to become pathogens. The lack of gene exchange from bacteriophages to Archaea may explain why so few (if any) archaeal pathogens exist (Lawrence, 2005; Bennewies et al., 2006; Gill and Brinkman, 2011).

Despite hypothesis discussed above Aminov (2013) from the Technical University of Denmark, National Veterinary Institute has published an article in which was stated role of Archaea in human disease. $\mathrm{He}$ looked into many potential clinical cases connecting with the Archaeal species. By him clarified that, patients with Crohn's disease, ulcerative colitis and primary pneumatosis intestinalis displayed a significantly lower incidence of methane excretion compared to healthy subjects. This work was the first suggesting an association of the Archaea with human gastrointestinal disease published in 1985. Since that time, there have been a number of studies, more recent ones using molecular ecology markers, which have confirmed these two initial observations (McKay et al., 1985; Aminov, 2013). Also Scanlan (2008) was written how the diarrheal conditions of human gastrointestinal disease result in the opposite trend with lower incidences of Methanogenic Archaea and lower rates of methane production. In addition to that, chemotherapy-induced diarrhea in cancer patients have also resulted in the decrease of Methanogenic Archaea in parallel with the loss of beneficial bacteria in 2013 (Scanlan et al., 2008).

Reassessments by many scientists show the role of methane production among patients with gastrointestinal disturbances have clearly associated the elevated methane production with alterations in intestinal motility, such as constipation, but not with other conditions. In this regard, alternative generation of highly toxic hydrogen sulfide. As a result, sulfate reduction in the gut may impose much higher health risks compared to more inert methane (Franck at al., 2012). 


\section{Archaea in oral cavity}

In another study, Lepp (2004) observed several clinical cases, which have been detected by PCR in up to $36 \%$ of periodontitis patients. So compared to periodontitis patients, the supragingival plaque of healthy subjects harbors a lower total microbial load, and the hydrogenotrophic group is represented exclusively by acetogenic bacteria at lower numbers too. On the other hand, the subgingival plaque from periodontitis patients harbors a larger number of total bacteria, and the hydrogenotrophic group includes methanogenic archaea and sulphate-reducing bacteria (SRB). The latter two groups are absent in healthy control subjects but present in $65 \%$ of periodontitis patients, alone or in combination (Lepp et al., 2004). However, the role of the Archaea in periodontal disease cannot be understand within the frames of a typical host-pathogen interaction, and it has to acknowledge that these are not bona fide pathogens (Bennewies et al., 2006; Liu et al., 2012).

Scientist states that, their involvement in disease can still be interpreted from the point of view of polymicrobial diseases that has recently gained considerable attention. Due to interface malfunction, a subset of usually symbiotic bacteria can display potentially pathogenic properties; they thus have been called "pathobionts" to be differentiated from the "classical," opportunistic pathogens. Then it was questioned if could the commensal methanogenic Archaea be considered as "pathobionts"? In polymicrobial diseases, such as periodontitis however, taxonomic signatures are less effective as disease predictors, although some attempts are being made to identify the key players within certain pathobiota (Chow et al., 2011).

The role of hydrogenotrophic microbiota is interchangeable and can be played by SRB, methanogenic Archaea, or acetogenic bacteria. Briefly, comparing of metagenomes of healthy and diseased microbiota may help to identify the sets of genes differentially represented in these two conditions and point to the enrichment or reduction of genes specific for pathologies. Signatures of periodontal disease indicate the enrichment by genes encoding metabolic functions that are consistent with a parasitic lifestyle and anaerobic metabolism, as well as by genes encoding virulence factors and the biosynthesis of toxic factors (Bartold et al., 2006; Liu et al., 2012; Aminov, 2013).

\section{Methanogenic archaea in diseases}

In a study by Convey and Makario (2008) were investigated an attitude of Methanogenic Archaea in health and disease. They collected many laboratory works and cases about Methanogens Archaea. For instance, it was suggested that we have to look at their pathogenicity from a various angle in comparison to classic pathogens that occupy tissues and release toxins. Instead, these organisms (methanogens) seem to share their pathogenicity indirectly, helping the growth of other microbes, which are directly involved in pathogenesis. Their data show that methanogens are more abundant in adults than in children (confirming results from other laboratories, discussed in their study). The study also shows that methanogens interact not only with SRB (sulphate-reducing bacteria) in the human and animal intestine but also with other bacteria. They presumed that, could it be that non-bacterial sources of $\mathrm{H} 2$ provide it for $\mathrm{H} 2$-using methanogens to grow? This is a possibility that deserves attention, for instance in colon cancer, in most of which M. smithii is more abundant than in healthy controls. Data from this study support also the concept that pathology of the abdominal aorta with impact on the colon vascular circulation is accompanied by a high incidence of breath-methane excretion. (Conway et al., 2008; Maczulak et al; 2000). Also reported that the frequency of methane breath excretors was upper among patients with malignant or pre-malignant colonic pathology, usually higher than in matched controls without colonic disease or with nonmalignant pathology. The same result was in colonic diverticulosis as well (Pique et al., 1984; Weaver et al., 1986).

Methanogens emerge in the intestinal tract of humans at about 2 years of age and then increase continuously with age reaching their highest concentrations in the elderly. This result was also, observed in the laboratory animals. It was obvious that the higher the percentage of methanogens in oral cavity, the more severe the periodontitis, and colon cancer is accompanied by a growth in colonic methanogen archaea (McKay et al., 1983).

Recently, a role for intestinal methanogens in obesity was noticed. Presence of methanogens in the colon contributes calorie and adiposis in laboratory mice, accordingly contributing to the progress of obesity. It was questioned if would M. smithii enhance growth or activity of the fiberconsumer polysaccharide-digesting bacteria and thus increase the utilization of fiber and caloric intake? If that were so, dietary fiber would be digested more efficiently and its caloric yield per unit weight would be greater than in the absence of M. smithii. It may also lead to aggregation of fat, obesity, especially in individuals who are on a high-fiber diet (Conway et al., 2008; Samuel and Gordon, 2006). 


\section{Archaea on skin}

There were several studies dedicated to the skin archaea although, there were not found much Archaea species. In a study by (Caporaso et al., 2011) periodically sampled the left and right palm of a male and female over few month, from the male was observed only transient Thaumarchaeota, while from the female observed persistent, albeit low, presence of these Archaea on her right palm. It has been stated that, different members of the Thaumarchaeota phylum are thought to be chemolithotrophic ammonium oxidizers and encode characteristic amoA gene homologs. Thus, people who sweat or exercise often could harbor larger communities of the Archaea. The scientists wrote also that, people use statin to control their cholesterol, thus they are cholesterol- lowering. However, at the same time this medical drug is anti - archaeal agent. That is why for today no exist exact information about how it would be harmful or beneficial for human microbiota disappearing Archaea Domain. Thus, some researchers suggest to think adequately before Archaea become part of "disappearing human microbiota" (Caporaso et al., 2011; Hulcr et al., 2012; Probst et al., 2013; Moissl-Eichinger et al., 2017).

Eckburg et al. (2003) published a study which was stated as "Archaea and Their Potential Role in Human Disease". They pointed about difficulties in the isolation and incubation of Archaea. Also, contribute to a relative lack of knowledge. It is puzzling that despite being one of the most numerous and ubiquitous life forms on earth, no member of the domain Archaea has been described as a human pathogen. These scientists tried to answer for this puzzle with its (archaea) cell structure difference. Archaea have ether-linked lipid and liposome (archaeosome) which play a role as potent immune adjuvants in vitro and in vivo. As an example, they inoculated an experimental vaccine to the mice. They vaccinated the mice with archaeasom which interrupted given listeriolysine. After several time, they noticed that this vaccination supposed prolonged specific immunity against Listeria monocytogenes. The explanation was like below: in immunology, archaeaosome activate APCs' by increasing expression of MHC class II and evoke strong antigen-specific responses to entrapped antigens when injected into the mice (Krishnan et al., 2000; Krishnan et al., 2001; Eckburg et al., 2003; Conlan et al., 2011).

\section{Effects of antibiotics on archaea}

According to some researches, utilizing of antibiotics may suppress the growth of some archaea. Upper levels of breath methane have been observed in patients with precancerous conditions and cancer of the colon than in healthy patients. A study by (Gijzen et al., 1991) suggests that antibiotics may effect to some archaea species. For example, "defaunation" of cockroaches with low concentrations of metronidazole results in a rapid drop in methane production, presumably due to methanogen eradication from the hindgut. It is unknown that these antibiotics directly kill methanogens in the gut, kill their ciliate protozoal hosts, or effect the local anaerobic bacterial population indirectly by altering the concentrations of coexistent methanogens. In a study from Spain a survey for antibiotic resistance within the genus Halobacterium showed that most extreme halophiles were resistant to lactams and aminoglycosides however were sensitive to many other antimicrobials, including macrolides, chloramphenicol, novobiocin, rifampin, bacitracin, and fluoroquinolones (Bonelo et al., 1984).

\section{Unsolved difficulties}

Currently, at least 16 Archaeal genome sequences have been studied. The completed genomes of Archaea, however, may still provide clues to the presence of possible virulence factors. For instance, a survey of genes that codify transcriptional regulators in four archaeal genomes revealed possible members of the bacterial Lys $R$ and sensor transduction regulator families. In addition, some of the members of these families are known to be related with controlling of virulence factors in bacterial pathogens. Unfortunately, there is no clear virulence phenotype as well as obvious animal model systems in which to estimate virulence. Methanogens are the only archaea that have been identified in humans. The default to identify other non-methanogens in humans might be in large part due to the lack of any molecular and biotechnological methods to define the abundance or diversity of archaea in human and animal microbiota. Methanogen Archaea may also follow virulence policy in eukaryotic organisms similar to those of the known anaerobic bacteria (Cavicchioli et al., 2003; Aminov, 2013).

Each scientist overviewed above conclude their studies about Archaea almost the same. No any clear or exact connection between archaea and disease has been clarified to date in human and animals, in part thanks to limitations in our ability to detect, identify, and isolate Archaea species. If Archaea are take part in human disease, it is likely that such participation will be illuminated using new molecular methods, knowing difficulties of their cultivation. When identified Archaea species the first time as a separate domain of taxonomy, its subspecies were dated as 
extremophiles and for these reasons unfamiliar to the human environment and microbiota. However, this discover was not a true reflection of archaeal physiology and we aware that their failure result emerge because of limited datasheets and narrow scientific opportunities. With the passing of days, molecular approaches have disclosed Archaea in decidedly non-extreme environments. It is hopeful that the appearance of application of similar and high techniques to the biology science may expand our perspective soon again. (Relman et al., 1990; Wilson et al., 1991; Relman et al., 1992).

\section{Conclusion}

No conclusive virulence genes or details have been described in Archaea to date. Nonetheless, Archaea

\section{References}

Aminov, R. I. (2013). Role of archaea in human disease. Frontiers in cellular and infection microbiology, 10.3389.

Balch, W. E., Fox, G. E., Magrum, L. J., Woese, C. R., \& Wolfe, R. S. (1979). Methanogens: reevaluation of a uniquebiological group. Microbiological reviews, 43, 260-296.

Bennewies T. T., Motro, Y., Hallin, P. F., \& Lund, O. (2006). Ten years of bacterial genome sequencing: comparative-genomics-based Functional and integrative genomics , 6(3) 165-185.

Bartold, P. M., \& Van Dyke, T. E. (2013). Periodontitis: a host-mediated disruption of microbial homeostasis. Unlearning learned concepts. Periodontology, 62, 203-217.

Bonelo, G., Ventosa., A. Megias., M. \& RuizBerraquero, F. (1984). The sensitivity of halobacteria to antibiotics. FEMS Microbiology letters, 21, 341345.

Cavicchioli, R., Curmi, P. M. G., Saunders, N., \& Thomas, T. (2003). Pathogenic archaea: do they exist? BioEssays, 25(11), 1119-1128.

Conlan, J. W., Krishnan, L. Willick., G. E. Patel.,G. B. \& Sprott, G. D. (2001). Immunization of mice with lipopeptide antigens encapsulated in novel liposomes prepared from the polar lipids of various Archaeobacteria elicits rapid and prolonged specific protective immunity against infection with the facultative intracellular pathogen Listeria monocytogenes. Vaccine, 19, 3509-3517.

Caporaso J.G., Lauber, C. L., Costello, E. K., Berg-Lyons, D., Gonzalez, A., Stombaugh, J., Knights, D., Gajer, P., Ravel, J., Fierer, N., Gordon, J. I., \& Knight, R. (2011). Moving pictures of the human may have the means, and they undouble have the chance, to cause disease. Whether or not members of the Archaea Domain possess virulence factors as commonly defined is questionable. Most scientist discussed human diseases in which archaea species may play a role as well as potential virulence characteristics of these organisms. Feasible elucidation for the current absence of information about Archaea as pathogens, and molecular methods that might be utilized in the search for such pathogens. Who knows it may discovered pathogenic Archaea after decade or nearly future accompanied by fresh molecular technologies and equipment in life sciences.

microbiome. Genome biology, 12(5), R50.

Chow, J., Tang, H., \& Mazmanian, K. S. (2011). Pathobionts of the gastrointestinal microbiota and inflammatory disease. Current opinion in immunology, 23(4), 473-480.

Conway de Macario, E., \& Macario, A. J. (2008). Methanogenic archaea in health and disease: a novel paradigm of microbial pathogenesis. International journal of medical microbiology, 299(2), 99-108.

Eckburg, P. B., Lepp, P. W., \& Relman, D. A. (2003). Archaea and their potential role in human disease. Infection and Immunity, 71(2), 591-596.

Carbonero, F., Benefiel, A. C., Alizadeh-Ghamsari, A. H., Gaskins, H. R. (2012). Microbial pathways in colonic sulfur metabolism and links with health and disease. Frontiers in physiology, 3, 448.

Gill E. E., \& Brinkman F. S. (2011). The proportional lack of archaeal pathogens: Do viruses/phages hold the key? BioEssays,33(4), 248-254.

Gijzen, H. J., Broers, C. A., Barughare, M. \& Stumm, C. K. (1991). Methanogenic bacteria as endosymbionts of the ciliate Nyctotherus ovalis in the cockroach hindgut. Applied and rnvironmental microbiology, 57, 1630-1634.

Hulcr, J., Latimer, A. M. Henley, J. B., Rountree.N. R., Fierer, N., Lucky, A., Lowman, M. D. \& Dunn R. R. (2012). A jungle in there: Bacteria in belly buttons are highly diverse, but predictable. Plosone 7, e47712.

Kandler, O., \& Konig H. (1998). Cell wall polymers in Archaea (Archaebacteria). Cellular and molecular life sciences, 54, 305-308. 
Krishnan, L., Dicaire, C. J., Patel, G. B., \& Sprott. G. D. (2000). Archaeosome vaccine adjuvants induce strong humoral, cell-mediated, and memory responses: comparison to conventional liposomes and alum. Infection and immunity, 68, 54-63.

Krishnan, L., Sad, S., Patel, G. B. \& Sprott, G. D. (2001). The potent adjuvant activity of archaeosomes correlates to the recruitment and activation of macrophages and dendritic cells in vivo. Journal of immunology, 166, 1885-1893.

Lawrence, J. G. (2005). Horizontal and vertical gene transfer: the life history of pathogens. Contributions to microbiology, 12, 255-271.

Lepp, P. W., Brinig, M. M., Ouverney, C. C., Palm, K., Armitage, G. C., \& Relman, D. (2004). Proceedings of the national academy of sciences, 101(16), 61766181.

Lewis, S., Cochrane, S. (2007). Alteration of sulfate and hydrogen metabolism in the human colon by changing intestinal transit rate. American journal of gastroenterology, 102, 624-633.

Liu, B., Faller, L. L., Klitgord, N., Mazumdar, V., Ghodsi M., Sommer, D. D., Gibbons, T. R., Treangen T. J., Chang, Y. C., Li, S., Stine, O. C., Hasturk, H., Kasif S., Segrè D., Pop, M., \& Amar, S. (2012). Deep sequencing of the oral microbiome reveals signatures of periodontal disease. PLoS one, 7 (6):e37919.

Martin, W. 2004. Pathogenic archaebacteria: do they not exist because archaebacteria use different vitamins? BioEssays, 26, 592-593.

McKay, L. F., Eastwood, M. A., Brydon, W. G. (1985). Methane excretion in man -a study of breath, flatus, and faeces. Gut 26, 69-74.

Madigan, M. T., Martinko, J. M. \& Parker J. (2000). Prokaryotic diversity: the Archaea, In M. T. Madigan, J. M. Martinko, and J. Parker (ed). Brock biology of microorganisms. p. 546-572. New Jearsy, US : Prentice-Hall, Inc.

Maczulak, A. E., Wolin, M. J., \& Miller, T. L. (1989). Increase incolonic methanogens and total anaerobes in aging rats. Applied and environmental microbiology, 55, 2468-2473.

McKay, L. F., Brydon, W. G., Eastwood, M. A., \&
Housley, E. (1983). The influence of peripheral vascular disease onmethanogenesis in man. Atherosclerosis, 47(1), 77-81.

Moissl-Eichinger, C., Probst, A. J., Birarda, G., Auerbach A, Koskinen, K., Wolf, P., \& Holman, H. N. (2017). Human age and skin physiology shape diversity and abundance of Archaea on skin. Scientific reports, 7 (1), 4039.

Piqué, J. M., Pallarés, M., Cuso', E., Vilar-Bonet, J., \& Gassull, M. A. (1984). Methane production and colon cancer. Gastroenterol, 87, 601-605.

Probst, A. J., Auerbach, A. K. \& Moissl-Eichinger, C. (2013). Archaea on human skin. PloS one 8, e65388.

Relman, D. A., Loutit, J. S. Schmidt, T. M. Falkow, S. \& Tompkins, L. S. (1990). The agent of bacillary angiomatosis. An approach to the identification of uncultured pathogens. New England journal of medicine, 323, 1573-1580.

Relman, D. A., Schmidt, T. M., MacDermott, R. P. \& S. Falkow (1992). Identification of the uncultured bacillus of Whipple's disease. New England journal of medicine, 327(5), 293-301.

Scanlan, P. D., Shanahan, F., \& Marchesi, J. R. (2008). Human methanogen diversity and incidence in healthy and diseased colonic groups using mcrA gene analysis. BMC microbiology, 20(8), 79.

Samuel, B. S., \& Gordon, J. I. (2006). A humanized gnotobiotic mouse model of host-archaeal-bacterial mutualism. Proceedings of the national academy of sciences, 103, 10011-10016.

Tseng, T. T., K. S. Gratwick., J. Kollman, D. Park., D. H. Nies., A. Goffeau., \& M. H. Saier, Jr. (1999).The RND permease superfamily: an ancient, ubiquitous and diverse family that includes human disease and development proteins. Journal of Molecular Microbiology and Biotechnology, 1(1), 107-125.

Weaver, G. A., Krause, J. A., Miller, T. L., \& Wolin, M. J. (1986). Incidence of methanogenic bacteria in a sigmoidoscopypopulation: an association of methanogenic bacteria anddiverticulosis. Gut, 27, 698-704.

Wilson, K. H., Blitchington, R., Frothingham, R. \& Wilson, J. A. (1991). Phylogeny of the Whipple'sdisease-associated bacterium. Lancet, 338, 474-475. 